[0212-7199 (2006) 23: 9; pp 441-445] ANALES DE MEDICINA INTERNA Copyright (C) 2006 ARAN EDICIONES, S.L.

AN. MED. INTERNA (Madrid) Vol. 23 , N. ${ }^{\circ}$, pp. $441-445,2006$

\title{
Intoxicación con litio
}

\section{DOMÍNGUEZ ORTEGA, O. MEDINA ORTIZ1, S. CABRERA GARCÍA- ARMENTER}

\author{
Servicio de Medicina Interna. Unidad de Medicina Familiar y Comunitaria y Unidad del \\ Sueño. Clínica Ruber. Madrid. ${ }^{I}$ Departamento de Psiquiatría. Unidad de Adolescentes. \\ Hospital General Gregorio Marañón. Madrid
}

\section{LITIUM INTOXICATION}

\begin{abstract}
RESUMEN
La intoxicación aguda por litio no es frecuente, entre el $75 \%$ al $90 \%$ de los pacientes con terapia de mantenimiento de litio pueden haber mantenido niveles tóxicos durante su tratamiento. La dosis por debajo de 2,5 $\mathrm{mmol} / \mathrm{L}$ no suelen asociarse a la aparición de síntomas. Los sistemas más afectados son el neurológico, gastrointestinal y cardiovascular. Los síntomas aparecen desde las dos y las cuatro horas postingesta. En las horas posteriores a la ingestión de litio, los niveles de litio no reflejan la severidad del cuadro clínico, no obstante la monitorización hormonal es importante para dirigir la agresividad terapéutica y marcar el pronóstico del cuadro. La utilización de forzar diuresis con solución salina isotópica, son mencionadas por diversos autores. Entre otras opciones también descritas está la diálisis.

En resumen, la intoxicación por litio puede ser un cuadro potencialmente frecuente y grave, por ello es necesario escribir unas normas de manejo terapéutico de esta entidad.
\end{abstract}

PALABRAS CLAVES: Litio. Intoxicación. Interacción medicamentosa. Diuréticos. Función renal.

\begin{abstract}
Litium intoxication has a low prevalence, the $75 \%$ of patients with treatment with litium could have toxic levels of litium during the treatment. Asyntomatic patients have been observed with doses under 2.5 $\mathrm{mmol} / \mathrm{L}$. Systems with high prevalence of symptoms are the nervious system, gastrointestinal tube and cardiovascular. Symptoms could appear from two hours to four hours after ingestion. Early hours after Litium ingestion, levels of litium are not related with severity. However, monitoring seric levels is necessary to lead therapeutic approach and to give a prognosis. Induce urine with isotonic solution, is used during therapeutic approach. Other therapeutic option is dialysis.

In summary, intoxication with litium could be a severe situation. It is necessary to describe therapeutic protocols in this entity.
\end{abstract}

KEY WORDS: Litium. Intoxication. Farmacologic interaction. Diuretic. Renal function.

Domínguez Ortega L, Medina Ortiz O, Cabrera García-Armenter S. Intoxicación con litio. An Med Interna (Madrid) 2006; 23: 441-445.

\section{INTRODUCCIÓN}

El litio, es uno de los fármacos clásicos, que ha demostrado desde hace varias décadas, ser efectivo en el control de la excitación que se produce en los pacientes con episodios maníacos $(1,2) \mathrm{y}$, en la prevención de nuevas crisis en los pacientes con trastorno bipolar (TB) $(3,4)$. Sin embargo, la intoxicación con litio es una complicación frecuente cuando se administra a largo plazo en pacientes con trastornos del estado del ánimo. Aún con pequeñas elevaciones de litio sérico, estos pacientes tienen una alta probabilidad de manifestar síntomas y signos de intoxicación grave comparados con pacientes que sufren una intoxicación aguda con niveles similares de litio en sangre, ya que, en una intoxicación aguda, los niveles de litio informados no se correlacionan directamente con los niveles en los tejidos, los cuales son mayores en pacientes que han recibido litio a largo plazo. Debido a esto, la toxicidad crónica se puede llegar a dar con bajas dosis de litio, en pacientes que llevan un tratamiento de varios años de evolución (5).

Desde 1949, cuando Cade (6,7), introdujo las sales de litio para el manejo de la manía, son varios los casos de intoxicación por litio que se han informado $(5,8,9)$. A mediados del siglo XIX, el litio era utilizado como la panacea para muchas afecciones comunes, por la comunidad médica, pero su alta toxicidad llevó a que el tratamiento con litio fuera prohibido en los Estados Unidos hasta 1970, cuando fue aprobado por la FDA (10) y, en 1974 se aprobó como terapia de mantenimien-

\footnotetext{
Trabajo aceptado: 19 de mayo de 2006
} 
to para pacientes con trastorno bipolar (TB) (11). Hoy en día, el litio sigue siendo utilizado como medicamento de elección para los episodios maníacos $(9,12)$, aun cuando continúen existiendo quienes están en su contra (13) y, algunos estudios muestren que aproximadamente entre el 75 al $90 \%$ de los pacientes con terapia de mantenimiento de litio pueden haber presentado niveles tóxicos durante su tratamiento (14).

Cuando un episodio maníaco llega a su fin, el riesgo de sufrir otro igual o un episodio depresivo es hasta de un $90 \%$ (15). La medicación clásica para prevenir este tipo de riesgo es el litio, pero aun cuando ha sido recomendada por diferentes guías de práctica médica (16), está visión está cambiando con el advenimiento de nuevos fármacos estabilizadores del humor, como la carbamacepina, valproato, lamotrigina, gabapentina, nimodipina y algunos antipsicóticos de nueva generación como la olanzapina (17). El advenimiento de estos fármacos en el tratamiento del TB ha traído consigo una disminución de su uso en los Estados Unidos en la última década (18). Sin embargo, en otros países como España, su utilización más bien se ha incrementado en los últimos 19 años, aun cuando es una medicación antigua que la industria ha dejado de publicitar desde hace ya tiempo y, su rango terapéutico está condicionado por una monitorización bastante exigente (19).

Además de controlar los episodios maníacos, el litio es utilizado en aquellos pacientes con depresión que no mejoran con los antidepresivos convencionales. Una gran variedad de estudios ha demostrado la eficacia de litio al ser asociado a aquellos pacientes con depresión, que no habían respondido o que lo habían hecho de forma parcial, cuando sólo estaban en tratamiento con antidepresivos (20).

\section{FARMACOLOGÍA}

El litio es un metal, que posee características similares al sodio y al potasio, pero con un tamaño más pequeño (8). Se administra a los pacientes como carbonato de litio y, una tableta de $300 \mathrm{mg}$ contiene $8,12 \mathrm{mEq}$ del ión litio. Después de su administración oral, la absorción comienza rápidamente y tarda aproximadamente 8 horas en completarse, presentando su mayor concentración en sangre entre la segunda y la cuarta hora (21). A menudo, este pico coincide con algunos efectos secundarios tempranos como trastornos gastrointestinales, pero al parecer los efectos secundarios que se presentan a largo plazo como temblor, aumento de peso y poliuria también se relacionan con estos picos (22). Su rango terapéutico varía según el procedimiento utilizado por el laboratorio, pero generalmente se encuentra entre 0,7 y $1,2 \mathrm{mEq} / 1$. La concentración de litio en el líquido cefaloraquideo es el $40 \%$ de su concentración en el plasma (23).

La medición de las drogas utilizadas en psiquiatría ha estado obstaculizada por la dificultad técnica de medir pequeñas cantidades de un fármaco en los diferentes fluidos corporales, sin embargo, el litio tiene la ventaja de ser un agente terapéutico activo que no se metaboliza y que puede ser fácilmente cuantificado, la concentración de litio en plasma es un indicador de la cantidad total de litio (11). Desde los años 50 se recomienda como rutina, la monitorización de los pacientes en tratamiento con litio (24) y en casos de intoxicación por litio, la monitorización continua es un requisito esencial (25).
Una dosis única de litio, tarda entre 12 y 27 horas en ser eliminada (26), pero esto varía con la edad. De 18 horas en un adulto joven a 36 horas en pacientes ancianos, aproximadamente. Al parecer, el tiempo de eliminación puede verse alterado por el tiempo de tratamiento, de tal manera, que un paciente que comienza con el tratamiento tardará menos tiempo en eliminar la droga de su organismo, que un paciente con una terapia de mantenimiento de un año o más (27).

La principal vía de eliminación del litio es la orina. Al no ser metabolizado y no encontrarse unido a proteínas, es filtrado libremente por los glomérulos. Aproximadamente el 95\% se excreta por el riñón pero se pueden encontrar algunas trazas en las heces (21). El 80\% del filtrado es reabsorbido junto con sodio y agua en los túbulos proximales, y el $20 \%$ restante se excreta en la orina . La aclaración renal de litio en un individuo normal debe estar entre 10 y $40 \mathrm{ml} / \mathrm{min}$ (28). Cualquier alteración en el funcionamiento renal puede repercutir directamente en la concentración de litio en sangre y, los mecanismos renales involucrados en la intoxicación por litio en pacientes sin enfermedad renal, se ven estrechamente ligados al balance del sodio (29). Por lo tanto, una deficiencia de sodio puede llevar a una disminución en la aclaración de litio (28). En un paciente con un adecuado balance electrolítico, el sodio y el litio son reabsorbidos en el túbulo próximal, el sodio puede ser reabsorbido también en el túbulo distal, particularidad que no posee el litio; pero en caso de una deficiencia extrema de sodio, el litio podría ser reabsorbido en el segmento distal de los túbulos. Si la deficiencia de sodio es moderada, la reabsorción de sodio que ocurre a nivel de los túbulos distales mantiene el balance, pero si la deficiencia de sodio es mayor, entonces ocurre una reabsorción de litio incrementando los niveles en plasma. Cuando las concentraciones de litio en plasma alcanzan cierto nivel, el litio inhibe la hormona aldosterona, lo que a su vez disminuye aún más la reabsorción de sodio en el túbulo distal; disminuyendo la aclaración de litio con un incremento aún mayor de litio en plasma. Se establece un círculo vicioso que sin una intervención terapéutica lleva a la intoxicación por litio (11).

Se ha descrito que el tratamiento crónico con litio causa algunos cambios en la estructura del riñón. Los estudios anatomopatológicos en los años 70 de algunos pacientes que habían sufrido intoxicación por litio, mostraban cambios morfológicos en sus riñones (30), lo que hacía pensar que estas alteraciones renales podían estar relacionadas con un tratamiento a largo plazo, que producían daño a nivel proximal y distal del nefrón. A comienzos de esta década, el estudio llevado a cabo por Bendz y cols., mostró que cuando los pacientes que han estado con un tratamiento crónico, dejan de tomar litio o disminuyen la dosis, la capacidad de concentración urinaria y la proporción de filtración glomerular no aumenta o, no se diferencia de quienes continúan el tratamiento con litio a dosis iguales que el inicio. La implicación clínica de este hallazgo consistiría en que a mayor tiempo de tratamiento mayor es la posibilidad de que ocurra una intoxicación por litio (31). Sin embargo, estudios más recientes, han revelado que el daño progresivo que ocurre a nivel de la función glomerular y tubular, podría estar relacionada más bien con otros factores como una intoxicación por litio ocurrida en algún momento del tratamiento, el mantenimiento de altos niveles de litio en plasma, interacciones medicamentosas, otras enfermedades somáticas y la edad, más que con el tiempo de tratamiento con litio, por lo que habría que controlar todas estas 
variables $(32,33)$. Estas dos opiniones nos muestran que la causa del daño renal durante el tratamiento con litio, es un punto que continúa generando controversia y no está nada claro en este momento.

\section{INTOXICACIÓN}

El litio es un ión altamente tóxico y, se han informado ya diversos casos de intoxicación en pacientes tratados con litio (29,34-36). La mayoría de las veces los síntomas son moderados, pero pueden ser indentificados por el médico. En casi todos los pacientes, la intoxicación se desarrolla de manera gradual con un predominio de los síntomas mentales y neurológicos.

El primer caso publicado de intoxicación por litio, ocurrió mucho antes de que se utilizara en psiquiatría. En un experimento llevado a cabo en si mismo, en 1913; Cleveland (37) describió por primera vez los síntomas neurológicos de una intoxicación aguda por litio, haciendo resaltar la ausencia de síntomas gastrointestinales.

La interacción con otras drogas también puede llevar a una alteración en los niveles de litio con la consecuente manifestación clínica. Se ha podido apreciar aumento en los niveles séricos de litio con el uso de algunos diuréticos. Las tiazidas disminuyen la aclaración de litio a nivel renal aumentando sus niveles. Otros estudios llevados a cabo en voluntarios sanos parecen sugerir que los diuréticos ahorradores de potasio, pueden elevar los niveles de litio en suero (11). También se ha informado sobre la intoxicación por litio en un paciente en quien se utilizó amiloride para el tratamiento de la insuficiencia cardiaca (38). Incluso, algunos diuréticos naturistas se han visto relacionados con un aumento en los niveles de litio de algunos pacientes que han tomado el producto con la intención de bajar de peso (39). Aun cuando los pacientes pueden mejorar tan solo con la retirada del litio y/o del diurético, se presenta a continuación un nuevo problema, ya que, los pacientes se muestran reticentes de volver a confiar nuevamente en un producto con el que se han sentido cerca de la muerte (38).

Otra medicación que se ha relacionado con un aumento de los niveles de litio en suero son los antinflamatorios como la indometacina (40), el piroxicam (41), diclofenato (42) y fenilbutazone (43), probablemente porque la inhibición de la síntesis de prostaglandinas disminuye la aclaración renal de litio. Pero por otro lado, no se ha visto esta alteración con el uso de la aspirina (44).

Además del aumento de los niveles de litio en sangre debido a las interacciones medicamentosas, también se pueden esperar reacciones adversas o complicaciones del cuadro médico al combinarlo con otras medicaciones de reciente aparición utilizadas en psiqiatríca. Ya comienzan a informarse efectos adversos por la posible combinación de litio y ziprazidona $(45,46)$ y litio con olanzapina $(47)$.

Un aumento en los niveles séricos de litio se corresponde casi siempre con la manifestación clínica de intoxicación, debido a esto, se propuso en 1978 una clasificación de intoxicación por litio basada en los niveles séricos de la droga (29). Los investigadores la subdividieron con los siguientes cortes: 1,5 a $2,5 \mathrm{mmol} / \mathrm{L}$ intoxicación moderada; 2,5 a 3,5 mmol/L intoxicación severa y más de 3,5 $\mathrm{mmol} / \mathrm{L}$ amenaza contra la vida. Pero los autores concluían que no se podía establecer una relación muy clara entre los puntos de corte y la severidad de los síntomas debido a que la literatura médica cita diversos casos de intoxicación con niveles normales de litio en suero y casos de niveles tóxicos sin manifestaciones clínicas $(48,49)$. Por lo que no se puede establecer el diagnóstico de intoxicación por litio solamente basados en los niveles séricos (50).

En muchas ocasiones, el diagnóstico clínico de la intoxicación por litio no es tan claro como parece, debido a la gran variedad de síntomas que se pueden presentar, sin embargo, la sintomatología neurológica es a menudo la que predomina (29). En la tabla I se muestran los síntomas y signos predominantes en la intoxicación por litio.

\section{TABLA I}

\begin{tabular}{ll} 
Sistema & Intoxicación por litio \\
\hline Nervioso central & Alteración de la conciencia, desde confusión hasta \\
& coma \\
& Síntomas cerebelosos: temblor, nistagmos, disar- \\
& tria, ataxia. \\
& Ganglios basales: movimientos coreónicos y/o par- \\
& kinsonianos \\
& Fatiga, apatía, hiperreflexia, desorientación, aluci- \\
& naciones \\
Gastrointestinal & Náuseas, vómito, diarrea \\
Cardiovascular & Síncope, cambios en la onda T, alteraciones en la \\
& conducción intraventricular \\
Renal & Poliuria, polidipsia, insuficiencia renal \\
Neuromuscular & Miopatía, neuropatía periférica \\
Endocrino & Hipotermia, hipertermia
\end{tabular}

Luego de una intoxicación por litio, la secuela más importante que se debe tener en cuenta es el Síndrome de Neurotoxicidad Irreversible por Litio (SIN-L), una alteración que se puede presentar aun con niveles terapéuticos de litio (51). Los síntomas predominantes están dados por disfunción persistente cerebelar, síndrome extrapiramidal persistente, disfunción persistente del tallo cerebral y demencia con diferentes grados de síndrome mental orgánico, pero también puede presentarse de manera atípica con mielinolisis pontina central y neuritis óptica retrobulbar (52). La etiología del SIN-L no está muy clara, pero al parecer, se relaciona con una amplia desmielinización de los nervios periféricos encontrada en las biopsias (53). Es probable que la desmielinización ocurra en múltiples lugares del sistema nervioso central, pero sobre todo a nivel del cerebelo, siendo la causa de las alteraciones neurológicas que persisten luego de la intoxicación, pérdida de las células de Purkinge, gliosis de la corteza cerebelar y atrofia cerebelar (52). El daño es irreversible y una vez que se produce es difícil un tratamiento con resultados satisfactorios. En algunos casos, existe una mejoría espontánea incluso completa, pero en la mayoría de los casos, las secuelas son permanentes. El tratamiento a largo plazo se basa en rehabilitación física para la ataxia, terapia lingüística para la disartria y entrenamiento cognitivo para la memoria y demencia (52). 


\section{ESTRATEGIAS TERAPÉUTICAS}

No parece existir ninguna evidencia clínica de que el lavado gástrico sea efectivo para el tratamiento de la intoxicación o sobredosis con litio (54).

En un principio es importante diferenciar si la intoxicación es crónica o aguda, es decir, si ocurre en un paciente que ha recibido tratamiento de manera prolongada y no ha tenido una ingestión aguda o, en un paciente que presenta la intoxicación luego de ser administrado el tratamiento, con o sin historia de tratamiento prolongado, ya que, existe diferencia entre la forma de presentación y el manejo. En el caso de una intoxicación crónica, todos los pacienten con síntomas que hagan pensar en una intoxicación por litio deberían ser hospitalizados y valorados por un nefrólogo para una posible diálisis, incluso aquellos cuyos niveles séricos de litio se encuentren en los rangos terapéuticos de 0,6 a 1,2 mmol/L. El cuidado debe ser prestado a cada paciente basado en los signos y síntomas clínicos de la intoxicación. Aquellos pacientes con criterios clínicos de intoxicación moderada a severa, son a menudo tratados en una unidad de cuidados intensivos (50).

La intoxicación aguda muchas veces es asintomática y las manifestaciones aparecen de manera retardada. La severidad de la intoxicación debe ser precisada con un interrogatorio detallado, determinando si es la primera vez, si ha sido por un intento suicida o si ha estado en tratamiento anteriormente $\mathrm{y}$, el paciente debe ser preferiblemente monitorizado. En muchas ocasiones se recomienda el tratamiento conservador hasta la aparición de un empeoramiento clínico (50).

La intoxicación por litio de manera deliberada, predomina sobre la intoxicación por razones terapéutica durante los primeros 3 años de iniciado el tratamiento, presentándose aproximadamente en el 77\% de los casos (55). Aun así, se ha podido apreciar que los pacientes con diagnóstico de trastorno bipolar en tratamiento con litio presentan una tasa de suicidio 3 veces menor que los que son tratados con otro estabilizador del ánimo, llegando a otorgársele propiedades antisuicidas (56).

La diálisis, continúa siendo el tratamiento de elección en la intoxicación por litio para conseguir una rápida disminución de los niveles de litio sérico. La hemodiálisis además contribuye a mejorar los niveles de conciencia y los síntomas neurológicos, aunque también se han utilizado en algunos casos diuréticos con buenos resultados (9).

\section{Bibliografía}

1. Gershon S. Lithium in mania. Clin Pharmacol Ther 1970; 11: 168-187.

2. Schou M. Forty years of lithium treatment. Arch Gen Psychiatry 1997; 54: 9-13; discussion 14-15.

3. Baastrup PC, Poulsen JC, Schou M, Thomsen K, Amdisen A. Prophylactic lithium: double blind discontinuation in manic-depressive and recurrent-depressive disorders. Lancet 1970; 2 (7668): 326-330.

4. Stallone F, Shelley E, Mendlewicz J, Fieve RR. The use of lithium in affective disorders. 3. A double-blind study of prophylaxis in bipolar illness. Am J Psychiatry 1973; 130: 1006-1010.

5. Nagappan R, Parkin WG, Holdsworth SR. Acute lithium intoxication. Anaesth Intensive Care 2002; 30: 90-92.

6. Cade JF. Med J Aust 1949; 2: 349-352.

7. Cade JF. Lithium salts in the treatment of psychotic excitement. 1949 Bull World Health Organ 2000; 78: 518-520.

8. Mark D, Okusa MD, Luz Jovita T, Crystal MD. Clinical Manifestations and Management of acute lithium Intoxication. The American Journal of Medicine 1994; 97: 383-389.

9. Eyer F, Pfab R, Felgenhauer N, Lutz J, Heemann U, Steimer W, Zondler S, Fichtl B, Zilker T. Lithium poisoning: pharmacokinetics and clearance during different therapeutic measures. J Clin Psychopharmacol 2006; 26: 325-330.

10. Strobusch AD, Jefferson JW. The checkered history of lithium in medicine. Pharm Hist 1980; 22: 72-76.

11. Cooper TB. Pharmacokinetics of lithium, in Psychopharmacology. The third generation of progress. Edited by Meltzar HY. New York, Raven Press, 1987. p. 1365-1375.

12. Goodwin FK. Rationale for long-term treatment of bipolar disorder and evidence for long-term lithium treatment. J Clin Psychiatry 2002; 63 (Supl. 10): 5-12.

13. Jefferson JW. Lithium. Still effective despite its detractors. BMJ 1998; 316 (7141): 1330-1331.

14. Amdisen A. Clinical features and management of lithium poisoning. Med Toxicol Adverse Drug Exp 1988; 3: 18-32.

15. Solomon DA, Keitner GI, Miller IW, Shea MT, Keller MB. Course of illness and maintenance treatments for patients with bipolar disorder. J Clin Psychiatry 1995; 56: 5-13.

16. Practice guideline for the treatment of patients with bipolar disorder. American Psychiatric Association. Am J Psychiatry 1994; 151 (12 Supl): $1-36$.

17. Kleindienst N, Greil W. Lithium in the long-term treatment of bipolar disorders. Eur Arch Psychiatry Clin Neurosci 2003; 253: 120-125.
18. Blanco C, Laje G, Olfson M, Marcus SC, Pincus HA. Trends in the treatment of bipolar disorder by outpatient psychiatrists. Am J Psychiatry 2002; 159: 1005-1010.

19. Castells X, Vallano A, Rigau D, Perez J, Casas M, Capella D. Trends in lithium prescription in Spain from 1985 to 2003. J Affect Disord 2006; 91: 273-276.

20. Bauer M, Forsthoff A, Baethge C, Adli M, Berghofer A, Dopfmer S, et al. Lithium augmentation therapy in refractory depression-update 2002. Eur Arch Psychiatry Clin Neurosci 2003; 253: 132-139.

21. Baldessarini RJ. Drugs and the treatment of psychiatric disorders, in The Pharmacological Basis of Therapeutics. Edited by Gillman AG, Goodman GS, Gillman A. Elmsford, New York, Pergamon Press; 1990. p. 383-435.

22. Schou M. in Principles of Psychopharmacology. 2nd ed. Edited by Clark W, del Guidice J. Chapter 17. New York, Academic Press, 1978.

23. Terhaag B, Scherber A, Schaps P, Winkler H. The distribution of lithium into cerebrospinal fluid, brain tissue and bile in man. Int J Clin Pharmacol Biopharm 1978; 16: 333-335.

24. Schou M. Lithium studies. 1. Toxicity. Acta Pharmacol Toxicol (Copenh) 1958; 15: 70-84.

25. Chen KP, Shen WW, Lu ML. Implication of serum concentration monitoring in patients with lithium intoxication. Psychiatry Clin Neurosci 2004; 58: 25-29.

26. Thornhill DP. Pharmacokinetics of ordinary and sustained-release lithium carbonate in manic patients after acute dosage. Eur J Clin Pharmacol 1978; 14: 267-271.

27. Goodnick PJ, Fieve RR, Meltzer HL, Dunner DL. Lithium elimination half-life and duration of therapy. Clin Pharmacol Ther 1981; 29: 47-50.

28. Thomsen K. Renal handling of lithium at non-toxic and toxic serum lithium levels. A review. Dan Med Bull 1978; 25: 106-115.

29. Hansen HE, Amdisen A. Lithium intoxication. (Report of 23 cases and review of 100 cases from the literature). Q J Med 1978; 47: 123-144.

30. Hestbech J, Hansen HE, Amdisen A, Olsen S. Chronic renal lesions following long-term treatment with lithium. Kidney Int 1977; 12: 205213.

31. Bendz H, Aurell M, Lanke J. A historical cohort study of kidney damage in long-term lithium patients: continued surveillance needed. Eur Psychiatry 2001; 16: 199-206.

32. Johnson G. Lithium--early development, toxicity, and renal function. Neuropsychopharmacology 1998; 19 (3): 200-205.

33. Lepkifker E, Sverdlik A, Iancu I, Ziv R, Segev S, Kotler M. Renal insuf- 
ficiency in long-term lithium treatment. J Clin Psychiatry 2004; 65: 850856

34. Lliteras M, Arilla A, Pérez J. Hemidiaphragmatic myoclonus during lithium intoxication. European Neuropsychopharmacology 2005; 15 (Supl. 3): S652.

35. Bilanakis N, Gibiriti M. Lithium intoxication, hypercalcemia and "accidentally" induced food and water aversion: a case report. Prog Neuropsychopharmacol Biol Psychiatry 2004; 28; 201-203.

36. Omata N, Murata T, Omori M, Wada Y. A patient with lithium intoxication developing at therapeutic serum lithium levels and persistent delirium after discontinuation of its administration. Gen Hosp Psychiatry 2003; 25: 53-55.

37. Ceveland S. A case of poisoning by lithium: presenting some new features. JAMA 1913; 60: 722

38. Macfie AC. Letter: Lithium poisoning precipitated by diuretics. Br Med J 1975; 1 (5956): 516

39. Pyevich D, Bogenschutx MP. Herbal diuretics and lithium toxicity. Am J Psychiatry 2001; 158: 1329.

40. Frolich JC, Leftwich R, Ragheb M, Oates JA, Reimann I, Buchanan D. Indomethacin increases plasma lithium. Br Med J 1979; 1 (6171): 11151116.

41. Kerry RJ, Owen G, Michaelson S. Possible toxic interaction between lithium and piroxicam. Lancet 1983; 1 (8321): 418-419.

42. Reimann IW, Frolich JC. Effects of diclofenac on lithium kinetics. Clin Pharmacol Ther 1981; 30: 348-352.

43. Singer L, Imbs JL, Schmidt M, Mack G, Sebban M, Danion JM. [The effects of phenylbutazone on the decrease of lithium clearance]. Encephale 1978; 4: 33-40

44. Reimann IW, Diener U, Frolich JC. Indomethacin but not aspirin increa- ses plasma lithium ion levels. Arch Gen Psychiatry 1983; 40: 283-286.

45. Miodownik C, Hausmann M, Frolova K, Lerner V. Lithium intoxication associated with intramuscular ziprasidone in schizoaffective patients. Clin Neuropharmacol 2005; 28: 295-297.

46. Borovicka MC, Bond LC, Gaughan KM. Ziprasidone- and lithium-induced neuroleptic malignant syndrome. Ann Pharmacother 2006; 40: 139142 .

47. Tuglu C, Erdogan E, Abay E. Delirium and extrapyramidal symptoms due to a lithium-olanzapine combination therapy: a case report. J Korean Med Sci 2005; 20: 691-694.

48. Speirs J, Hirsch SR. Severe lithium toxicity with "normal" serum concentrations. Br Med J 1978; 1 (6116): 815-816.

49. Kondziela JR. Extreme lithium intoxication without severe symptoms Hosp Community Psychiatry 1984; 35: 727-728.

50. Sadosty AT, Groleau GA, Atcherson MM. The use of lithium levels in the emergency department. J Emerg Med 1999; 17: 887-891.

51. Johnson GF. Lithium neurotoxicity. Aust N Z J Psychiatry 1976; 10: 33 38.

52. Adityanjee, Munshi KR, Thampy A. The syndrome of irreversible lithium-effectuated neurotoxicity. Clin Neuropharmacol 2005; 28: 38-49.

53. Uchigata M, Tanabe H, Hasue I, Kurihara M. Peripheral neuropathy due to lithium intoxication. Ann Neurol 1981; 9: 414

54. Teece S, Crawford I. Best evidence topic report: no clinical evidence for gastric lavage in lithium overdose. Emerg Med J 2005; 22: 43-44.

55. Montagnon F, Said S, Lepine JP. Lithium: poisonings and suicide prevention. Eur Psychiatry 2002; 17: 92-95.

56. Goodwin FK, Fireman B, Simon GE, Hunkeler EM, Lee J, Revicki D Suicide risk in bipolar disorder during treatment with lithium and divalproex. Jama 2003; 290: 1467-1473. 